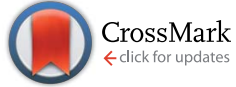

Cite this: J. Mater. Chem. A, 2014, 2 , 18076

Received 16th July 2014

Accepted 9th September 2014

DOI: $10.1039 / c 4 t a 03646 h$

www.rsc.org/MaterialsA

\section{Decomposition synthesis of tuneable, macroporous carbon foams from crystalline precursors via in situ templating $\dagger$}

\author{
D. Ressnig, ${ }^{\star a}$ T. Corbiere, ${ }^{a}$ T. Lunkenbein, ${ }^{b}$ U. Braun, ${ }^{c}$ M. G. Willinger ${ }^{b}$ \\ and M. Antonietti ${ }^{a}$
}

\begin{abstract}
A flexible, sustainable, one-step thermal decomposition route for the synthesis of hierarchical, heteroatom doped carbon foams is presented. Task-specific semi-organic crystals combine functions for three different purposes: the carbon and heteroatom source, a foaming agent $\left(\mathrm{CO}_{2}\right)$ and an in situ generable template $(\mathrm{NaCl})$. Insights to the decomposition pathway were gained through FTIR/MS coupled TGA and an ultrafast out-of-furnace heating procedure and the products were analysed with (HR)SEM/TEM, EELS, FTIR, and $\mathrm{N}_{2}$ sorption. The resulting macroporous carbon foams are excellent supports for metallic nanoparticles due to their hierarchical structure, high surface area and tuneable heteroatom contents. This was demonstrated for catalytically active copper or the magnetic CoNi alloy for water purification.
\end{abstract}

\section{Introduction}

Heteroatom doped (O, N, S) carbons are ubiquitous and display promising applications in different fields including hydrogen storage, ${ }^{1}$ catalysis, ${ }^{2}$ supercapacitors, ${ }^{1 b, 3}$ or sensors, ${ }^{4}$ owing to their high structural and chemical versatility. As macroporous materials, they are excellent supports for heterophase catalysts due to their tuneable surface acidity/basicity, wettability, electrical properties, unhindered reactant flow and in particular high thermal and chemical stability. ${ }^{5}$ The presence of heteroatoms in carbon based support materials is of crucial importance for the post-synthetic functionalization with metal/metal oxide nanoparticles through wet-chemical processes. Localized electron density and topological irregularities provide welldispersed anchor sites for metal cations. Purely graphitic carbons in contrast need typically to be treated with harsh oxidants prior to functionalization with similar procedures. ${ }^{5}$

The design of monolithic carbons is attractive from the perspectives of straightforward processing, device implementation and material recycling. Flexible and conceptually simple templating methods have been successfully established for the preparation of monolithic, well-ordered carbons during

\footnotetext{
${ }^{a}$ Max Planck Institute of Colloids and Interfaces, Am Mühlenberg 1, 14476 Potsdam, Germany.E-mail: Debora.Ressnig@mpikg.mpg.de

${ }^{b}$ Fritz Haber Institute of the Max Planck Society, Department of Inorganic Chemistry, Faradayweg 4-6, 14195 Berlin, Germany

'Bundesanstalt für Materialforschung und Prüfung, Unter den Eichen 87, 12205 Berlin, Germany

$\dagger$ Electronic supplementary information (ESI) available: SEM images for $\mathrm{NaAc}-\mathrm{Cl}$ derived pristine and functionalized foams and nitrogen sorption isotherms. FTIR/MS coupled TGA analyses of the thermal decomposition of NaAc-Cl. See DOI: $10.1039 / \mathrm{c} 4 \mathrm{ta} 03646 \mathrm{~h}$
}

the past decades. ${ }^{6}$ However, practical drawbacks reduce the incentive to follow these routes, given the laborious template preparation and post-synthesis etching steps that bear the risk to destroy the materials's microstructure, which in particular holds for heteroatom doped carbons.

A simplified alternative profits from the in situ formation of hard templates, generated through the decomposition of metal organic precursor complexes. The metal rich templates such as $\mathrm{MgO},{ }^{7}$ also in combination with $\mathrm{Mo},{ }^{8} \mathrm{CaC}_{2} / \mathrm{SrC}_{2},{ }^{9}$ or $\mathrm{Fe}_{3} \mathrm{C},{ }^{10}$ come with the advantage that they act as both moulds and catalysts for graphitization of external carbon sources at elevated temperatures. Graphitization improves the electrical conductivity but typically reduces the amount of structural heteroatoms and often produces a mixture of different nanostructures including carbon cages and nanotubes. ${ }^{7}$ The graphitic shell and the inherently low reactivity of these templates against most etching agents moreover hinders their removal significantly and prolongs etching procedures, which likely damage the material and influence its physicochemical properties. Nevertheless, in situ templating is a remarkably simple approach and finding non-graphitizing, water soluble templates is a crucial next step. We here introduce a straightforward, flexible method for the synthesis of macroporous carbons that relies on the in situ precipitation of $\mathrm{NaCl}$ crystallites during the thermal decomposition of semi-organic, crystalline precursors. Alkali halide salts come with the clear advantages over other hard templates of being low-cost and easily removable by simple washing with water, hence ecologically and economically friendly. Furthermore, salt fluxes, pure or as eutectic mixtures, have recently emerged as powerful porogens in the synthesis of meso- and microporous carbons. ${ }^{\mathbf{1 1}}$ Our method covers the macroporous range in this cascade. 
The thermal decomposition of solid precursors currently emerges as a simple top-down pathway to access structured bulk materials. ${ }^{12}$ These transformations are mainly of topological nature, where significant amounts of the initial parent crystals are evolved as gaseous decomposition products leaving a porous bulk behind. Most of the prepared materials are oxideceramics that find widespread applications owing to their favourable, often also hierarchical, structuration. ${ }^{\mathbf{1 3}}$ The solid state polymerizations of halogenated acrylates to polyesters build another class of thermally induced topological transformations. The polymerization goes in hand with intercalation of metal halide crystals. This however is an excellent starting point to synthesise macroporous carbons via in situ templating. To do so, we conceptually extend the thermal polymerization to a decomposition approach, to access tuneable, continuous, macroporous carbon foams. Heteroatom doping is easily achieved through the right choice of the precursor crystals. Their intrinsically good wettability further allows the direct functionalization with metal nanoparticles via wet-impregnation techniques.

\section{Experimental}

\section{Synthetic procedures}

Sodium-2-chloropyridine-3-carboxylate. 2-Chloropyridine-3carboxylic acid (1.57 g, $10 \mathrm{mmol}$ ) was dissolved in a mixture of acetone $(80 \mathrm{~mL})$ and $\mathrm{H}_{2} \mathrm{O}(2 \mathrm{~mL})$ with stirring at RT. Subsequently, a solution of $\mathrm{NaOH}(400 \mathrm{mg}, 10 \mathrm{mmol})$ dissolved in $\mathrm{H}_{2} \mathrm{O}(2 \mathrm{~mL})$ was added and the mixture was strongly stirred for $10 \mathrm{~min}$. Crystallization was induced from the resting solution through slow solvent evaporation at RT. The colourless crystals were washed with cold acetone and dried in vacuo at $50{ }^{\circ} \mathrm{C}$. The formation of the carboxylate was confirmed by FTIR analysis and the product was obtained with a yield of $1.8 \mathrm{~g}, 100 \%$.

Thermal decomposition of NaAc-Cl, NaPyr-Cl and CT. The precursor salts sodium-2-monochloroacetate ( $\mathrm{NaAc}-\mathrm{Cl}$ ), sodium 2-chloropyridine-3-carboxylate (NaPyr-Cl) or chloramine- $\mathrm{T}$ (CT) (200 $\mathrm{mg}$ to $1 \mathrm{~g}$ ) were homogenized with a mortar prior to the thermal treatment. The thermal decomposition was performed in a Nabertherm muffle furnace by heating the powders to $550{ }^{\circ} \mathrm{C}$ (rate $10 \mathrm{~K} \mathrm{~min}^{-1}$ ) for $30 \mathrm{~min}$ under a constant nitrogen flow (15 $\mathrm{L} \mathrm{min}^{-1}$ ). The monolithic products were washed overnight with DI $\mathrm{H}_{2} \mathrm{O}$ after using sonication for $10 \mathrm{~min}$ and subsequently dried at $80{ }^{\circ} \mathrm{C}$ in air.

For the fast, out-of-furnace heating, a heat gun setup as described previously was used. ${ }^{14}$ The powders were filled to a quartz tube and directly heated to $430{ }^{\circ} \mathrm{C}$ under nitrogen flow $\left(0.4 \mathrm{~L} \mathrm{~min}^{-1}\right)$ with a total reaction time of $10 \mathrm{~min}$.

For the thermal post treatment, the washed carbons were heated at a rate of $5 \mathrm{~K} \mathrm{~min}^{-1}$ to $1200{ }^{\circ} \mathrm{C}$ under a nitrogen flow $\left(15 \mathrm{~L} \mathrm{~min}^{-1}\right)$ in a Nabertherm tube furnace for $3 \mathrm{~h}$.

Functionalization of NaAc-Cl. Homogenized $\mathrm{NaAc}-\mathrm{Cl}$ decomposition products (15 mg) were impregnated with $3 \mathrm{~mL}$ of a $0.3 \mathrm{M}$ aqueous solution of $\mathrm{Cu}\left(\mathrm{NO}_{3}\right)_{2}$ or a mixture of $\mathrm{Co}\left(\mathrm{NO}_{3}\right)_{2}$ and $\mathrm{Ni}\left(\mathrm{NO}_{3}\right)_{2}(1: 1)$ for $1 \mathrm{~h}$ with magnetic stirring. The supernatant was removed by filtration and the solid was dried at $80{ }^{\circ} \mathrm{C}$ in air. The powders were subsequently heated to $600{ }^{\circ} \mathrm{C}$ $\left(10 \mathrm{~K} \mathrm{~min}^{-1}\right)$ under a nitrogen flow $\left(15 \mathrm{~L} \mathrm{~min}^{-1}\right)$ and directly cooled.

Catalytic reduction of $\boldsymbol{p}$-nitrophenol. The catalyst $(5 \mathrm{mg})$ was dispersed in DI $\mathrm{H}_{2} \mathrm{O}(10 \mathrm{~mL})$ by sonication, before $(0.25 \mathrm{~mL}$, $0.025 \mathrm{mg}$ catalyst) of the dispersion was transferred to a solution of $p$-nitrophenol $\left(0.7510^{-4} \mathrm{M}, 50 \mathrm{~mL}\right)$ using a micropipette. The suspension was stirred at $150 \mathrm{rpm}$ at RT. After addition of $\mathrm{NaBH}_{4}(95.0 \mathrm{mg}, 2.5 \mathrm{mmol}, 0.05 \mathrm{M}$ ) aliquots of $1.5 \mathrm{~mL}$ were removed at an interval of $1 \mathrm{~min}$ and directly analysed by UV-vis spectroscopy.

\section{Characterization methods}

FTIR spectra were collected in ATR geometry on a VARIAN 1000 spectrometer, Scimitar Series (FTS 1000). Elemental analysis was performed with an Elementar vario MICRO cube. Powder Xray diffraction patterns were measured with copper $\mathrm{K} \alpha(1.54 \AA)$ radiation on a Bruker D-8 device, equipped with a scintillation detector in reflection mode. Morphology observations and EDX measurements were performed on a JEOL (JSM - 7500 F) instrument, equipped with an X-MAX $80 \mathrm{~mm}^{2}$ detector from Oxford Instruments. Nitrogen sorption isotherms were recorded using a Quantachrome Quadrasorb SI porosimeter with $\mathrm{N}_{2}$ at $77 \mathrm{~K}$, after degassing at $150{ }^{\circ} \mathrm{C}$ for 20 hours. Absorption branch points $\left(p / p_{0}<0.1\right)$ were applied in the BET model to calculate the apparent surface area.

Thermogravimetric measurements were performed on a Netzsch thermal analysis device TG 209 F1 under a flow of nitrogen and with a heating rate of $10 \mathrm{~K} \mathrm{~min}^{-1}$. Transmission electron microscopy (TEM) and electron energy loss spectroscopy (EELS) were recorded on a Cs corrected FEI Titan 80-300 with an acceleration voltage of $80 \mathrm{kV}$ and $300 \mathrm{kV}$. No beam induced damage of the sample was observed during measurements.

\section{Results and discussion}

For the one-step preparation of a porous carbon from an ionic crystal, the precursor must generally include a metal cation and an organic, carbonizable residue that is functionalized with the anion of the target porogen. Sodium monochloroacetate (NaAc$\mathrm{Cl}$ ) is one of the simplest of these systems as it consists of sodium and chloride as template cation and anion respectively, separated by an acetate residue as the minimal organic subunit (Fig. 1). Our other precursors, sodium-2-chloropyridine-3carboxylate (NaPyr-Cl) and chloramine-T (CT) are in contrast rich in $\mathrm{sp}^{2}$ carbon and provide heteroatoms $(\mathrm{S}, \mathrm{N})$ for direct in situ doping (Fig. 1). NaAc-Cl and CT are low cost and commercially available, while NaPyr-Cl was prepared by the deprotonation of acetic acid with $\mathrm{NaOH}$ in a water/acetone mixture, followed by slow crystallization ( $c f$. experimental section).

The finely-crushed precursor powders were heated at a rate of $10 \mathrm{~K} \mathrm{~min}^{-1}$ to $550{ }^{\circ} \mathrm{C}$ for $30 \mathrm{~min}$ under an inert nitrogen atmosphere for the decomposition synthesis. The three corresponding thermogravimetric analysis (TGA) curves are compared in Fig. 1. For NaAc-Cl a single, but pronounced weight loss sets in at around $240{ }^{\circ} \mathrm{C}(46 \mathrm{wt} \%)$. For NaPyr-Cl, a 


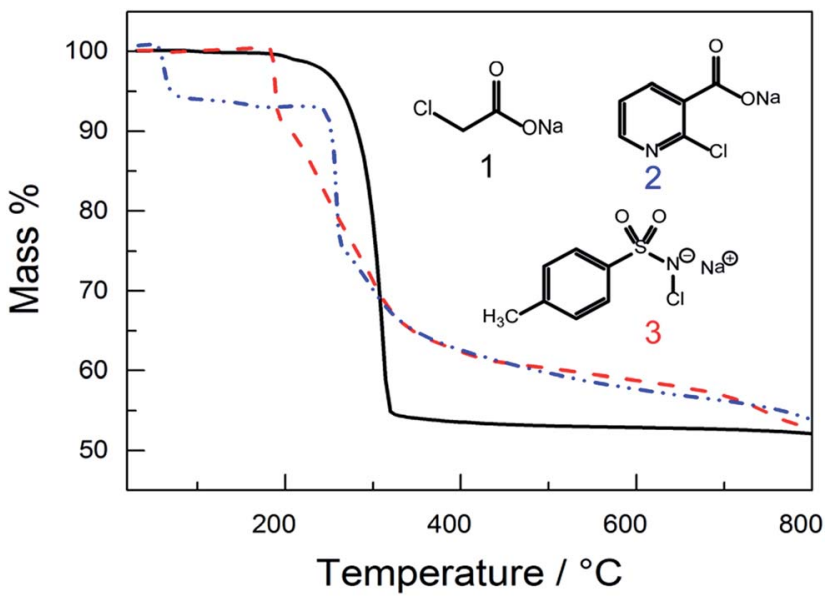

Fig. 1 TGA curves, recorded with $10 \mathrm{~K} \mathrm{~min}^{-1}$ under a flow of $\mathrm{N}_{2}$, track the thermal decomposition of sodium-2-monochloroacetate (1 $\mathrm{NaAc}-\mathrm{Cl}$ ), sodium-2-chloropyridine-3-carboxylate (2 $\mathrm{NaPyr}-\mathrm{Cl}$ ) and $\mathrm{N}$-chloro- $\mathrm{p}$-toluenesulfonamide sodium salt (3 CT).

pre-step starts at $55{ }^{\circ} \mathrm{C}(6 \mathrm{wt} \%)$, which corresponds to evaporating acetone (b.p. $58^{\circ} \mathrm{C}$ ) that remains from the deprotonation/ crystallization reaction. A pronounced weight loss sets in at $274{ }^{\circ} \mathrm{C}$ (35 wt\%), while CT starts to decompose at $185{ }^{\circ} \mathrm{C}$ (41 wt\%). The mass remaining at $550{ }^{\circ} \mathrm{C}$ (i.e. after subtraction of $\mathrm{NaCl}$ ) is higher for NaPyr-Cl (28 wt\%) and CT (34 wt\%) products compared to NaAc-Cl (4 wt\%), consistent with the molecular structure of the precursors.
The general synthetic procedure is scalable and the inset in Fig. 2a shows the product of $20 \mathrm{~g}$ NaAc-Cl precursor. The initial mass was reduced by $95 \%$ to $0.79 \mathrm{~g}$ after decomposition and removal of $\mathrm{NaCl}$ by repeated washing with water. All three precursors yield stable, easily processable monoliths, which are truncated with pores in the milli- micrometre ranges. Scanning electron microscopy (SEM) images show that the monoliths possesses hierarchical properties as removal of $\mathrm{NaCl}$ generates cubic shaped pores in the micro- nanometre ranges. This implies that the in situ generated $\mathrm{NaCl}$ crystals are replicated as hard endo-templates and excludes significant intermediate formation processes in a salt melt, which would lead to the creation percolation networks with smaller, less defined porosity. ${ }^{\mathbf{1 1}}$ The typical edge length of the cavities depend on the starting compounds and range from $200 \mathrm{~nm}$ up to approximately $2 \mu \mathrm{m}$ for $\mathrm{NaAc}-\mathrm{Cl}$, from $80 \mathrm{~nm}$ to $250 \mathrm{~nm}$ for $\mathrm{NaPyr}-\mathrm{Cl}$ and from $100 \mathrm{~nm}$ to $350 \mathrm{~nm}$ for CT. Faster decomposition - as described below - prohibits the diffusion and growth of $\mathrm{NaCl}$ crystallites by immediate inclusion to the carbon matrix and hence, leads to formation of smaller more uniform cubic voids of $0.5 \mu \mathrm{m}$ to $1 \mu \mathrm{m}$ for NaAc-Cl (Fig. S1 $\dagger$ ). The thickness of carbon scaffold is defined by the ratio of template to organic residue in the precursor compounds. The finest features are therefore obtained from NaAc-Cl with a width of $10 \mathrm{~nm}$ to $25 \mathrm{~nm}$, as it consists of just one available carbon atom per $\mathrm{NaCl}$ unit. The decomposition of $\mathrm{NaAc}-\mathrm{Cl}$ was additionally executed by applying an ultrafast (10 $\mathrm{min}$ ) out-of-furnace heating technique that can be visually followed ( $c f$. experimental section). It was observed that the precursor passes a liquid stage during the

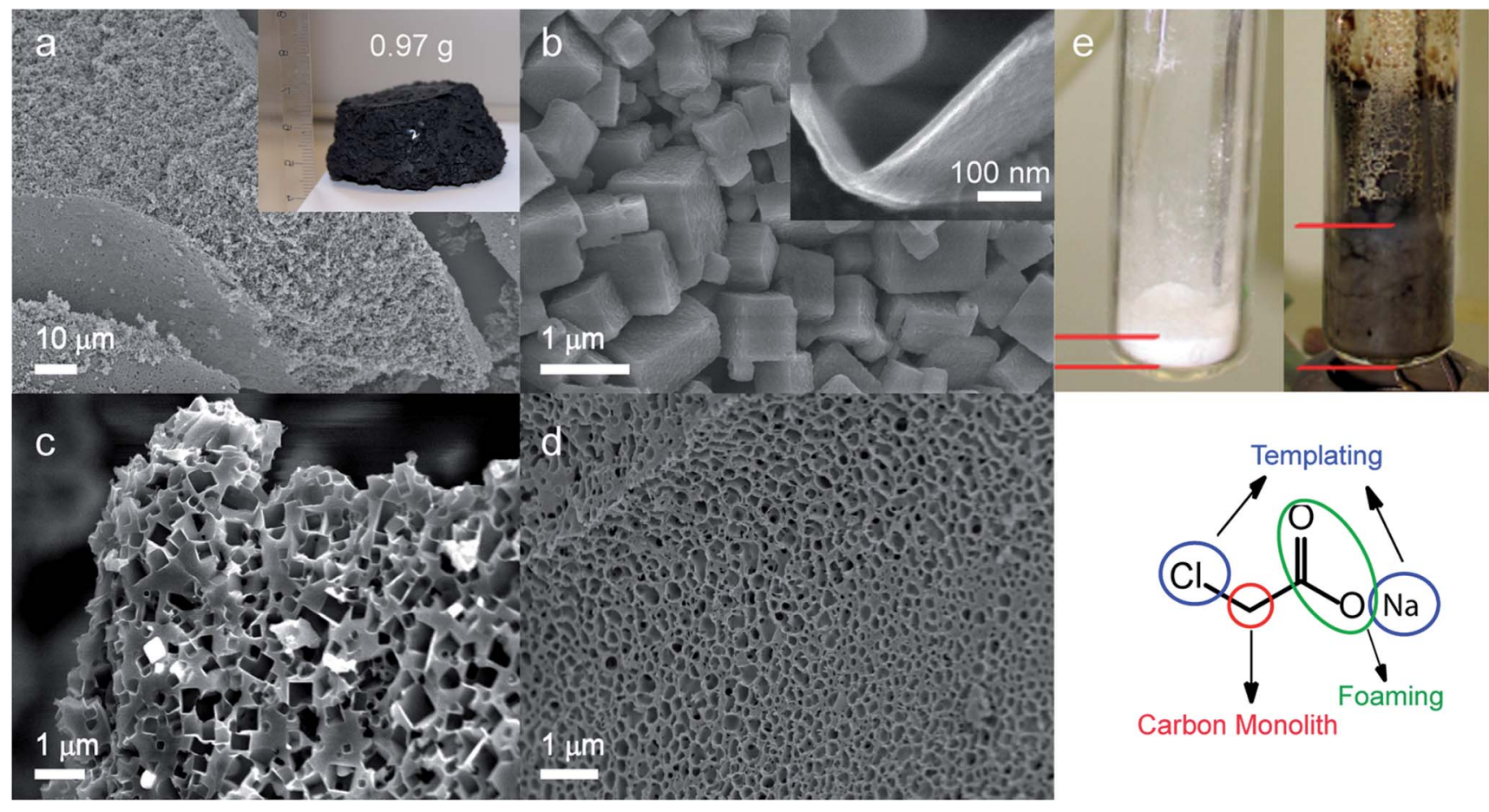

Fig. 2 SEM images of thermally decomposed (a and b) NaAc-Cl, (c) NaPyr-Cl and (d) CT. The inset in (a) shows a macroscopic foam synthesised from $20 \mathrm{~g} \mathrm{NaAc}-\mathrm{Cl}$. (e) Photograph of $\mathrm{NaAc}-\mathrm{Cl}$ powders before and after the thermal decomposition with the heat-gun setup illustrate the drastic volume expansion. Functions of different precursor moieties are described at the example of $\mathrm{NaAc}-\mathrm{Cl}$. 
transformation, which may occur just locally along the reaction front or within the entire reactant volume depending on the amount of material. The subsequent carbonization goes in hand with a drastic volume expansion due to the evolution of volatile products (Fig. 2e).

The volatile decomposition products of $\mathrm{NaAc}-\mathrm{Cl}$ were analysed with FTIR or MS coupled TGA (Fig. S2 and S3†). The mass change from the decomposition step at around $300{ }^{\circ} \mathrm{C}$ can be attributed to the loss of mainly carbon dioxide and water according to MS. FTIR further indicates formation of acetic acid and other hydrocarbon components, likely small condensed molecular units. The above data suggest that the nucleation of $\mathrm{NaCl}$, which is thermodynamically favoured, occurs quickly after reaching a certain temperature threshold and in a direct solid-solid fashion. The precipitation of $\mathrm{NaCl}$ forces the remaining organic fragments to a liquid state, thus allowing the formation of continuous monoliths. These highly reactive organic fragments undergo polymerization, dehydration and decarboxylation reactions to form the resulting monolithic carbons within just seconds. The evolution of volatile by-products has an additional foaming effect to generate hierarchic structures. The majority of the evolved gas is $\mathrm{CO}_{2}$, which is a well-known industrial foaming agent. ${ }^{15} \mathrm{~A}$ carbon/template/ foaming agent ratio of $(1 / 1 / 1)$ appears to be favourable to the formation of light weight monoliths with an ultrathin scaffold structure.

The carbon foam derived from NaAc-Cl features an apparent surface area $S_{\mathrm{BET}}$ of $483 \mathrm{~m}^{2} \mathrm{~g}^{-1}$ with a total pore volume of 0.57 $\mathrm{cm}^{2} \mathrm{~g}^{-1}$, which can be attributed to the contribution of micropores and mesopores with a broad size distribution (Fig. S3 $\dagger$ ). This surface area is twice as high as for example carbon aerogels synthesized by hydrothermal routes. ${ }^{16}$ The apparent $S_{\mathrm{BET}}$ surface areas of NaPyr-Cl $\left(15 \mathrm{~m}^{2} \mathrm{~g}^{-1}\right)$ and CT $\left(8 \mathrm{~m}^{2} \mathrm{~g}^{-1}\right)$ are significantly lower.

The FTIR spectra give insight to remaining functional groups after carbonization (Fig. 3). The absence of aromatic or aliphatic $\mathrm{C}-\mathrm{H}$ stretches in the region of $3000 \mathrm{~cm}^{-1}$ points to a high degree

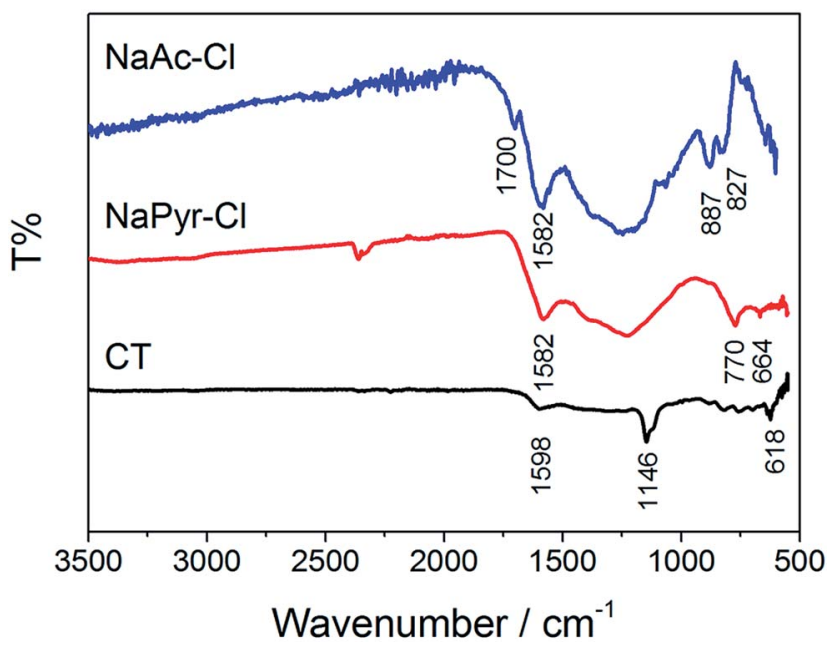

Fig. 3 FTIR spectra of carbons derived from different semi-organic precursor crystals. of aromatization. The band at $1702 \mathrm{~cm}^{-1}(\mathrm{C}=\mathrm{O}$ stretch $)$ is relatively weak for $\mathrm{NaAc}-\mathrm{Cl}$, and even absent for $\mathrm{NaPyr}-\mathrm{Cl}$, indicating that most of the carbonyl functionalities were eliminated in the course of the reaction. The pronounced band at $1590-1580 \mathrm{~cm}^{-1}$ is present in all three spectra and typical for aromatic $(\mathrm{C}=\mathrm{C})$ valences. The regions in-between $1500 \mathrm{~cm}^{-1}$ and $1050 \mathrm{~cm}^{-1}$ correspond to an overlap of vibrations from (hetero-)aromatic systems with alkoxy $(\mathrm{C}-\mathrm{O}-\mathrm{C})$ groups, and the sharp bands $<1000 \mathrm{~cm}^{-1}$ are characteristic for the fingerprint region of aromatics. ${ }^{17}$ The band at $1146 \mathrm{~cm}^{-1}$ in the CT spectrum corresponds to sulfonamide groups $\left(-\mathrm{SO}_{2} \mathrm{~N}\right)$ and consequently the absorptions between $770 \mathrm{~cm}^{-1}$ and $618 \mathrm{~cm}^{-1}$ to $(\mathrm{S}-\mathrm{N})$ and $(\mathrm{S}-\mathrm{C})$ vibrations. ${ }^{18}$

The composition of the different foams was deduced by elemental analysis (Table 1) and identifies carbon as major component. Negligible amounts of hydrogen are present in $\mathrm{NaAc}-\mathrm{Cl}$ foams and $3 \mathrm{wt} \%$ remain in NaPyr-Cl or CT products. The contents of oxygen heteroatoms range above $10 \mathrm{wt} \%$. Nitrogen and sulphur could also be successfully incorporated to the foams. The nitrogen/carbon ratio in NaPyr-Cl is 0.2 , which is equal to the value of pure pyridine pointing towards high stability of the aromatic ring structure during the decomposition. Also the $\left(-\mathrm{SO}_{2}-\right)$ unit appears to remain intact according to the constant atomic ratio of oxygen to sulfur and FTIR results.

Further modifications will be discussed in depth for the for $\mathrm{NaAc}-\mathrm{Cl}$ derived foams as representative system. The surface polarity of the carbon monoliths was switched from hydrophilic to hydrophobic through elimination of the oxygen groups by thermal post-treatment (Fig. 4a). The oxygen content was thereby reduced from $15 \mathrm{wt} \%$ to $<5 \mathrm{wt} \%$ (Table 1). Restructuration through loss of oxygen affects the texture of the material and reduces the apparent surface area from $483 \mathrm{~m}^{2} \mathrm{~g}^{-1}$ to 296 $\mathrm{m}^{2} \mathrm{~g}^{-1}$, whilst maintaining the cubic porous structure (Fig. $4 \mathrm{~b}$ and S5 $\dagger$ ). The long and short-range connectivity motifs of carbon are essential for defining the electronic properties, which becomes more relevant for the thermally reduced carbons. The atomic binding state was evaluated by electron energy loss spectroscopy (EELS) (Fig. 4c). The EELS spectra display energy loss peaks at $286 \mathrm{eV}$ and $294 \mathrm{eV}$ that are characteristic of the $1 \mathrm{~s}$ to $\pi^{*}$ and $\sigma^{*}$ transitions of $\mathrm{sp}^{2}$ hybridized carbon. ${ }^{19}$ The relative strength of the $\pi^{*}$ and the high $\pi^{*}$ to $\sigma^{*}$ ratio further verifies the predominance of graphitic bound carbon..$^{20}$ No long-range atomic order can be determined from HRTEM analyses, as expected from fast polymerizing fragments (Fig. 4d). Nevertheless, a pattern of prolated structures is indicative for the formation of small graphitic platelets. Moreover, the selected area diffraction pattern (SAED) shows two

Table 1 Elemental composition of different, heteroatom doped carbon foams

\begin{tabular}{lccccr}
\hline [Wt\%] & $\mathrm{C}$ & $\mathrm{N}$ & $\mathrm{H}$ & $\mathrm{S}$ & $\mathrm{O}$ \\
\hline NaAc-Cl 550 & 79 & $<1$ & 1 & $<1$ & 15 \\
NaAc-Cl 1200 & 95 & $<1$ & $<1$ & $<1$ & 5 \\
NaPyr-Cl & 70 & 14 & 3 & $<1$ & 13 \\
CT & 63 & 7.5 & 3.5 & 11 & 14
\end{tabular}




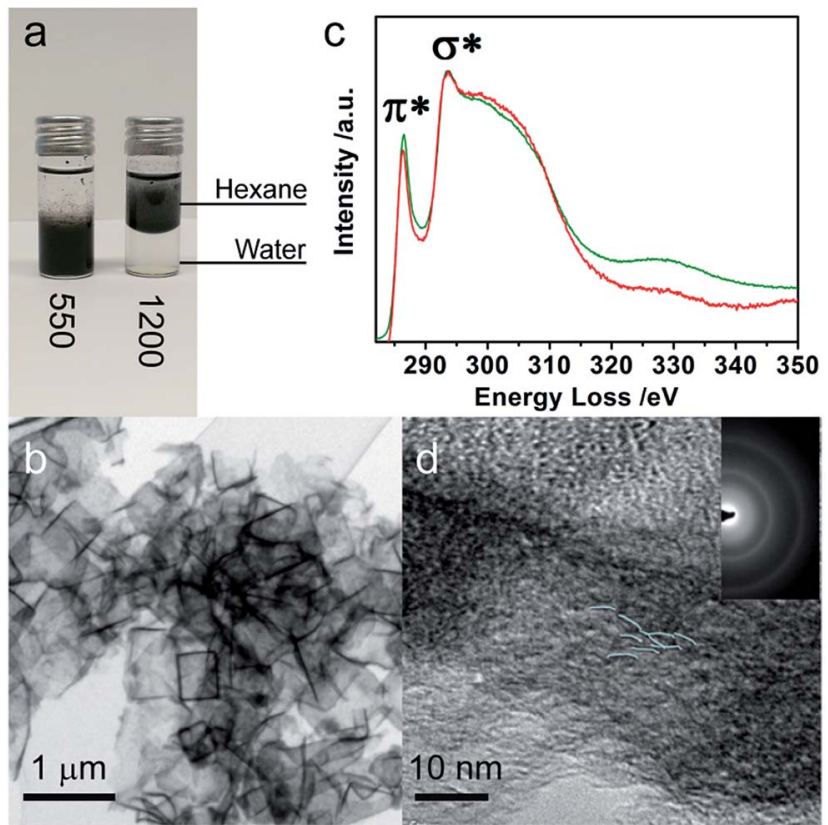

Fig. 4 (a) The surface polarity is switched from polar to apolar by thermal post treatment at $1200^{\circ} \mathrm{C}$. (b) TEM analysis indicates that the cubic pore shape is retained in the thermal post treatment. (c) Two representative EELS spectra of post-treated foams depict the predominance of $\mathrm{sp}^{2}$ configured carbon. (d) HRTEM analysis shows atomic orientation into small graphitic platelets with no significant long range order. The inset shows the corresponding SAED pattern.

diffuse cycles that reflect the basal and edge plain stacking distances of graphite, pointing towards minimal short range atomic order.

The high sensitivity of the thin carbon layers unfortunately does not allow for the collection of meaningful Raman spectra for further structure elucidation. The electronic conductivity of carbon foams is a function of a complex interplay of structural and density sensitive factors and typically affected by meso- and macroporosity. ${ }^{21}$ The conductivity was evaluated with impedance spectroscopy (1-1000 Hz) using a two electrode setup and accounts for the thermally post-treated samples $0.85 \mathrm{~S} \mathrm{~m}^{-1}$ (graphitic powder, experimental: $2.66 \mathrm{~S} \mathrm{~m}^{-1}$ ).

\section{Functionalization}

The carbons were functionalized by a wet-chemical procedure through impregnation with aqueous solutions of $\mathrm{Cu}\left(\mathrm{NO}_{3}\right)_{2}$ or $\mathrm{Co} / \mathrm{Ni}\left(\mathrm{NO}_{3}\right)_{2}(0.3 \mathrm{M})$. The presence of oxygen heteroatoms is essential to the wettability and cation adsorption, hence defining the resultant degree of dispersion of the metal nanoparticles. Reduction of the metal cations was achieved by thermal treatment at $600{ }^{\circ} \mathrm{C}$. Carbon materials feature the advantage over other supports (e.g. zeolites or metal oxides), that they act as both reductant and stabilizers for the metal nanoparticles. ${ }^{22}$ HRSEM and TEM analyses confirm that metallic particles are homogeneously dispersed over the entire carbon foam (Fig. 5). The CoNi alloy forms particles of approximately $20 \mathrm{~nm}$ in diameter. The powder X-ray diffraction (PXRD) reflections are shifted versus the references of pristine
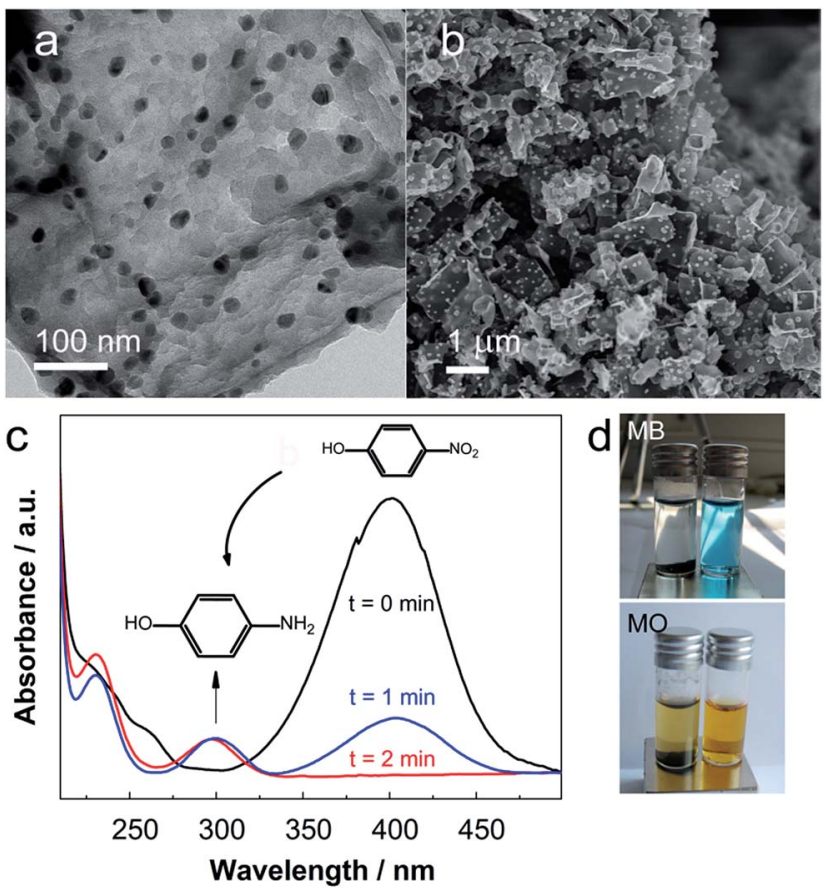

Fig. 5 (a) TEM image of a CoNi/C composite. (b) SEM image of a $\mathrm{Cu} / \mathrm{C}$ composite. (c) The UV-vis spectra track the reduction of $p$-nitrophenolate catalysed by $\mathrm{Cu} / \mathrm{C}$. (d) Adsorption of methylene blue (MB) or methyl orange (MO) to $\mathrm{CoNi} / \mathrm{C}$. The photographs compare pristine solutions (right) with solution after dye adsorption and magnetic separation of the adsorbent (left).

Co or Ni to intermediate $2 \theta$ positions, indicative for the adaption of the crystal lattice in accordance to formation of an alloy (Fig. S6 $\dagger$ ). The deposited copper particles show a larger size variation between approximately $50 \mathrm{~nm}$ and $100 \mathrm{~nm}$ (Fig. 5b). Also here, PXRD reflections can be clearly assigned to metallic copper (Fig. S6†).

The catalytic activity and accessibility of the deposited copper nanoparticles was approved with the catalytic reduction of $p$-nitrophenolate in the presence of $\mathrm{NaBH}_{4} \cdot{ }^{23}$ The transformation can be easily tracked with UV-vis spectroscopy by following the disappearance of the absorption band at $400 \mathrm{~nm}$ and appearance of the band at $300 \mathrm{~nm}$ corresponding to $p$ aminophenolate (Fig. 4c). The hydrophilic character of the oxygen functionalized carbon is here not only an advantage but rather essential, to disperse the composite in water.

Magnetic carbon composites have great potential for application in different fields, e.g. as magnetic toners, as ferrofluids, magnetic resonance imaging or for environmental issues. ${ }^{24}$ Water purification is a major concern due to the increased pharmaceutical waste that accumulates in the water cycle and drastically disturbs ecological systems. ${ }^{25}$

In a proof of principle we demonstrate adsorption of organic dyes as waste model systems. The CoNi modified carbons adsorb pollutants of different polarities, with predominance for cationic species (Fig. 4d). Knowing that macropores are dominant, which are characteristically not molecule traps, it can be assumed that surface defects and composition are relevant for 
the adsorption of the dyes. The NiCo/C monoliths can be easily recovered through magnetic separation.

\section{Conclusions}

We present a versatile, one-step route to synthesize heteroatom rich, hierarchical macroporous carbon foams via the thermal decomposition of semi-organic crystals without the need for external templates. The key is that the precursor crystals are functionalized for three main purposes: (1) with the carbon and heteroatom source, (2) with carboxylate that generates $\mathrm{CO}_{2}$ as foaming agent, (3) with a chloride group and a sodium cation as in situ generable template $(\mathrm{NaCl})$. Phase separation, i.e. nucleation of $\mathrm{NaCl}$, is thermodynamically favoured and allows carbonization at comparably low temperatures, which offers optimal conditions to maintain structural heteroatoms. The amounts of heteroatoms can therefore be adjusted through thermal post-treatments. We further demonstrate straightforward functionalization with metal nanoparticles and achieve high dispersion due to the presence of oxygen in the pristine carbon foam. The metal/carbon composites may be used for different purposes from which catalysis and waste water treatments are just two options.

The absolute porosity of the foams as well as mechanical properties remain to be elucidated with regards to specific applications. Moreover, there is still much more room to investigate other crystalline precursors e.g. through variation of the alkali halide or organic unit to obtain products with different structures, morphologies and properties.

\section{Notes and references}

1 (a) Y. Xia, Z. Yang and Y. Zhu, J. Mater. Chem. A, 2013, 1, 9365; (b) M. Sevilla and R. Mokaya, Energy Environ. Sci., 2014, 7, 1250.

2 (a) X.-K. Kong, C.-L. Chen and Q.-W. Chen, Chem. Soc. Rev., 2014, 43, 2841; (b) T.-P. Fellinger, F. Hasché, P. Strasser and M. Antonietti, J. Am. Chem. Soc., 2012, 134, 4072.

3 G. Wang, L. Zhang and J. Zhang, Chem. Soc. Rev., 2012, 41, 797.

4 (a) S. Peng and K. Cho, Nano Lett., 2003, 3, 513; (b) F. Villalpando-Páez, A. H. Romero, E. Muñoz-Sandoval, L. M. Martınez, H. Terrones and M. Terrones, Chem. Phys. Lett., 2004, 386, 137.

5 J. Zhu, A. Holmen and D. Chen, ChemCatChem, 2013, 5, 378. 6 (a) H. Nishihara and T. Kyotani, Adv. Mater., 2012, 24, 4473; (b) A. H. Lu and F. Schüth, Adv. Mater., 2006, 18, 1793; (c)
A. Thomas, F. Goettmann and M. Antonietti, Chem. Mater., 2008, 20, 738.

7 (a) K. Xie, X. Qin, X. Wang, Y. Wang, H. Tao, Q. Wu, L. Yang and Z. Hu, Adv. Mater., 2012, 24, 347; (b) S. Chen, J. Bi, Y. Zhao, L. Yang, C. Zhang, Y. Ma, Q. Wu, X. Wang and Z. Hu, Adv. Mater., 2012, 24, 5593.

8 M. Motiei, J. Calderon-Moreno and A. Gedanken, Adv. Mater., 2002, 14, 1169.

9 Y. Saito and T. Matsumoto, Nature, 1998, 392, 237.

10 J. N. Wang, L. Zhang, J. J. Niu, F. Yu, Z. M. Sheng, Y. Z. Zhao, H. Chang and C. Pak, Chem. Mater., 2007, 19, 453.

11 (a) N. Fechler, T.-P. Fellinger and M. Antonietti, Adv. Mater., 2013, 25, 75; (b) X. Liu, N. Fechler and M. Antonietti, Chem. Soc. Rev., 2013, 42, 8237.

12 H.-D. Yu, M. D. Regulacio, E. Ye and M.-Y. Han, Chem. Soc. Rev., 2013, 42, 6006.

13 (a) O. Herzberg and M. Epple, Eur. J. Inorg. Chem., 2001, 1395; (b) M. Epple and O. Herzberg, J. Mater. Chem., 1997, 7, 1037.

14 D. Ressnig, G. Clavel, N. Scharnagl and M. Antonietti, Part. Part. Syst. Charact., 2014, 31, 557.

15 L. J. M. Jacobs, M. F. Kemmere and J. T. F. Keurentjes, Green Chem., 2008, 10, 731.

16 S.-A. Wohlgemuth, R. J. White, M.-G. Willinger, M.-M. Titirici and M. Antonietti, Green Chem., 2012, 14, 1515.

17 T. Hartono, S. Wang, Q. Ma and Z. Zhu, J. Colloid Interface Sci., 2009, 333, 114.

18 C.-O. Argelia, M.-R. Christian, O.-S. Fabián, M.-H. Sheila, B.-G. Virgilio and R. Gildardo, Quim. Nova, 2011, 34, 787.

19 A. Thomas, A. Fischer, F. Goettmann, M. Antonietti, J.-O. Müller, R. Schlögl and J. M. Carlsson, J. Mater. Chem., 2008, 18, 4893.

20 K. A. Katrinak, P. Rez and P. R. Buseck, Environ. Sci. Technol., 1992, 26, 1967.

21 N. Amini, PhD Thesis, University of London, 2010.

22 (a) J. Zhu, A. Holmen and D. Chen, ChemCatChem, 2013, 5, 378; (b) G. Chieffi, C. Giordano, M. Antonietti and D. Esposito, J. Mater. Chem. A, 2014, 2, 11591; (c) J. M. Campelo, D. Luna, R. Luque, J. M. Marinas and A. A. Romero, ChemSusChem, 2009, 2, 18.

23 P. Herves, M. Perez-Lorenzo, L. M. Liz-Marzan, J. Dzubiella, Y. Lu and M. Ballauff, Chem. Soc. Rev., 2012, 51, 5577.

24 M. Zhu and G. Diao, Nanoscale, 2011, 3, 2748.

25 (a) D. G. J. Larsson, Upsala J. Med. Sci., 2014, 119, 108; (b) S. Kaplan, Crit. Rev. Environ. Sci. Technol., 2013, 43, 1074. 